\title{
The Natural History of a Missed Scaphoid Fracture
}

\author{
Gijsbert D. Musters ${ }^{\mathrm{a}}$, Karine M. van Scherpenzeel ${ }^{\mathrm{b}}$, Simon D. Strackee ${ }^{\mathrm{c}}$, J. Carel Goslings ${ }^{\mathrm{a}, \mathrm{d}}$
}

\begin{abstract}
Scaphoid fractures are known to be a difficult diagnosis since they can be accompanied by unspecific physical complaints. Furthermore, they are difficult to visualize on standard $\mathrm{x}$-ray examinations. The purpose of this report is to stress the importance of adequate diagnosis and treatment of scaphoid fractures. We describe a 50-year old female, with progressive wrist pain after a skiing accident, who was diagnosed with an untreated scaphoid fracture. This scaphoid fracture had developed into a scaphoid non-union advanced collapse of the wrist. The scaphoid non-union advanced collapse was treated with a four-corner arthrodesis and a Wafer procedure, which ultimately led to an acceptable situation. This report presents a rare complication of a missed scaphoid fracture. X-rays of the wrist commonly fail to demonstrate fractures of the scaphoid bone. Therefore more invasive imaging techniques must be used to diagnose a scaphoid fracture.
\end{abstract}

Keywords: Scaphoid; Fracture; Non-union; Diagnosis; SNAC wrist

\section{Introduction}

The diagnosis of scaphoid fractures is known to be difficult since they can be accompanied by unspecific physical

\footnotetext{
Manuscript accepted for publication August 23, 2011

aAcademic Medical Center, Trauma Unit, Department of Surgery, Amsterdam, The Netherlands

${ }^{\mathrm{b} C h a r i t e}$ University Hospital, Department of Trauma Surgery and Orthopaedics, Berlin, Germany

cAcademic Medical Center, Department of Plastic and Reconstructive Surgery, Amsterdam, The Netherlands

${ }^{\mathrm{d}}$ Corresponding author: J. Carel Goslings,

Email: j.c.goslings@amc.nl
}

doi:10.4021/jmc287w complaints [1]. Furthermore, scaphoid fractures can be difficult to visualize on standard X-rays [2]. If suspicion of a scaphoid fracture has risen, further evaluation may be warranted. This is supported by the fact that scaphoid fractures relatively commonly lead to non-union due to the limited vascularization of the scaphoid bone. Therefore, additional imaging such as MRI, bone scintigraphy or CT scanning is frequently used in order not to miss the diagnosis $[2,3]$. Treatment consists of prolonged immobilization or osteosynthesis and screw fixation.

However, the literature on this topic is scarce, especially in addressing the late results of untreated scaphoid fractures. Therefore the following case report was started. This case report clearly shows the late results of an untreated scaphoid fracture and stresses the importance of adequate diagnosis and treatment.

\section{Case Report}

A 50-year old female presented nine months after a skiing accident with progressive pain at her left wrist. Initial

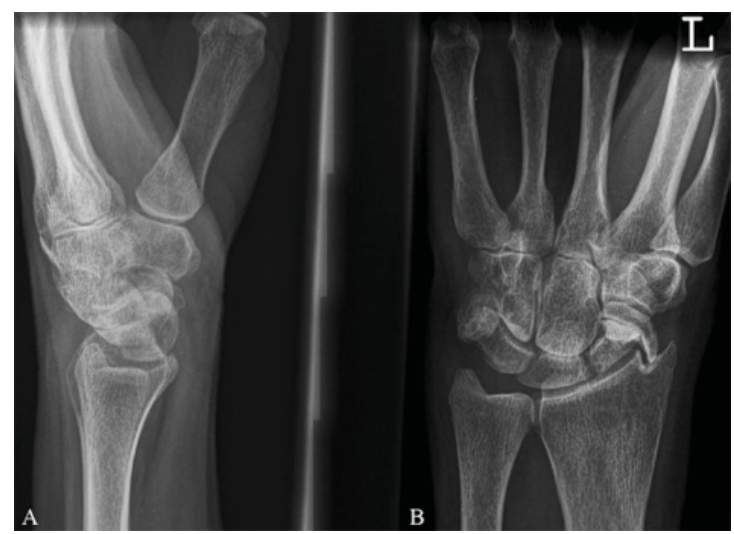

Figure 1. A: Lateral view of the left wrist. B: Anterior-posterior view of the left wrist. $A$ and $B$ demonstrate a hook shaped pseudarthrosis of the scaphoid, a deep irregularity of the distal radius articular surface due to erosion by the distal scaphoid fragment, and collapse of the carpus. 
examinations did not raise suspicion of a missed scaphoid fracture. One year after the accident the X-rays of the wrist showed a hook shaped pseudarthrosis of the scaphoid, a deep irregularity of the distal radius articular surface due to erosion by the distal scaphoid fragment, and collapse of the carpus, matching a scaphoid non-union advanced collapse (SNAC) of the wrist (Fig. 1). The SNAC wrist was treated with a four-corner arthrodesis. After the arthrodesis the patient developed a type 1 Complex Regional Pain Syndrome for which a Wafer procedure was warranted. Immediately after the procedure the pain of the wrist was reduced. Due to the limited mobilization a contracture of the fifth finger developed. The patient was treated with intensive physiotherapy, occupational therapy and psychotherapy, which led to a reduction in the contracture of the fifth finger and a reduction of the pain. However, the movement restrictions remained in the fifth finger. The patient was prescribed a knuckle bender splint on her fourth and fifth finger when participating in activities such as work, sports and driving. The loading capacity of the wrist is still increasing while the usage of the splint is decreasing.

\section{Discussion}

This report shows, to our knowledge for the first time, a rather complex complication of a natural history of an untreated scaphoid fracture. Due to the limited literature little is known about the natural history of the scaphoid fracture.

Scaphoid fractures can be accompanied by unspecific physical complaints [1]. Standard X-rays of the wrist fail to demonstrate a fracture in up to $50 \%$ of the patients [4]. Therefore it is likely to encounter an untreated scaphoid fracture. The increased use of CT or MRI scans in the evaluation of wrist pain might enhance this phenomenon [6]. Thus confronting surgeons with a difficult call to make: how should the encountered untreated scaphoid fracture be treated?

Immobilization in the presence of clinical suspicion, repeated Roentgen exams or additional imaging techniques are necessary to prevent further damage $[4,5]$. The incidence of pseudarthrosis in untreated fractures is unknown; if treated by immobilization it varies from $4 \%$ to $12 \%[1,4]$. The initial dislocation of the fracture and the resulting carpal collapse correspond to the degree of degenerative defects to which a pseudarthrosis will lead. Therefore, an operative treatment with reduction and stable fixation of a scaphoid pseudarthrosis is advised [2].

We conclude that X-rays of the wrist commonly fail to demonstrate fractures of the scaphoid bone. Therefore more invasive imaging techniques should be used to diagnose a scaphoid fracture. If such a fracture is found, operative treatment is advised to prevent its later complications. To prevent these kinds of complications early recognition and treatment are essential.

\section{Source of Funding}

The authors declare that they have no competing interest.

\section{References}

1. Bohler L, Trojan E, Jahna H. The results of treatment of 734 fresh, simple fractures of the scaphoid. J Hand Surg Br. 2003;28(4):319-331.

2. Mack GR, Bosse MJ, Gelberman RH, Yu E. The natural history of scaphoid non-union. J Bone Joint Surg Am. 1984;66(4):504-509.

3. Mallee W, Doornberg JN, Ring D, van Dijk CN, Maas M, Goslings JC. Comparison of CT and MRI for diagnosis of suspected scaphoid fractures. J Bone Joint Surg Am. 2011;93(1):20-28.

4. Dorsay TA, Major NM, Helms CA. Cost-effectiveness of immediate MR imaging versus traditional follow-up for revealing radiographically occult scaphoid fractures. AJR Am J Roentgenol. 2001;177(6):1257-1263.

5. Kawamura K, Chung KC. Treatment of scaphoid fractures and nonunions. J Hand Surg Am. 2008;33(6):988997.

6. Dias JJ, Wildin CJ, Bhowal B, Thompson JR. Should acute scaphoid fractures be fixed? A randomized controlled trial. J Bone Joint Surg Am. 2005;87(10):21602168. 